\title{
Phase transitions in anisotropic superconducting and magnetic systems with vector order parameter: three-loop renormalization group analysis
}

\author{
S. A. Antonenko and A. I. Sokolov \\ Department of Physical Electronics, Electrotechnical University, \\ Prof.Popov Str. 5, St.-Petersburg, 197376, Russia
}

The critical behavior of the model with $N$-vector complex order parameter and three quartic coupling constants which describes phase transitions in unconventional superconductors, helical magnets, stacked triangular antiferromagnets, superfluid helium-3 and zero-temperature transitions in fully frustrated Josephson-junction arrays is studied within the field-theoretical renormalization group (RG) approach in three dimensions. To obtain qualitatively and quantitatively correct results perturbative expansions for $\beta$-functions and critical exponents are calculated up to three-loop order and resummed by means of the generalized Padé-Borel procedure. Fixed point coordinates, critical exponents values, RG flows etc. are found for physically interesting cases $N=2$ and $N=3$. Critical (marginal) values of $N$ at which the topology of the flow diagram changes are determined as well. It is argued, on the base of several independent criterions, that the accuracy of the numerical results obtained is about 0.01 , an order of magnitude better than that given by resummed two-loop RG expansions.

In most cases the systems mentioned are shown to undergo fluctuation-driven first-order phase transitions. Continuous transitions are allowed in hexagonal $d-$ wave superconductors, in planar helical magnets (into sinusoidal linearly-polarized phase), and in triangular antiferromagnets (into simple unfrustrated ordered states) 
with critical exponents $\gamma=1.336, \nu=0.677, \alpha=-0.030, \beta=0.347, \eta=0.026$ which are hardly believed to be experimentally distinguishable from those of the $3 D X Y$ model. The chiral fixed point of RG equations is found to really exist and possess some domain of attraction provided $N \geq 4$. So, magnets with Heisenberg $(N=3)$ and $X Y$-like $(N=2)$ spins would not demonstrate chiral critical behavior with unusual values of critical exponents; they can approach the chiral state only via first-order phase transitions.

64.60.A, 74.65.+n, 75.40.Cx, 75.50.R

Typeset Using REVTEX 


\section{INTRODUCTION}

The renormalization group $(\mathrm{RG})$ approach in three dimensions proved to be very efficient when used to study the critical behavior of simple $O(n)$-symmetric models. Calculations of higher-order RG expansions for field-theoretical $\beta$-functions and critical exponents combined with proper resummation of the series obtained resulted in the estimates of critical exponent values which nowadays are referred to as most accurate (canonical) numbers [1,2]. This approach enabled one to give quantitatively correct description of critical behavior of more complex systems possessing two quartic coupling constants in their Landau-Wilson Hamiltonians. We mean the impure Ising model [3] 5], the cubic model [4, 6], and the $m n^{-}$ model [7]. Moreover, the method turned out to be powerful enough even in two dimensions as was shown by comparison of the approximate results obtained on the base of four-loop RG expansions resummed by means of Padé-Borel-like technique with their exact counterparts known for (exactly solvable) $2 D$ Ising and impure Ising models [1,22,5].

On the other hand, there are numerous models with more than two quartic coupling constants which describe phase transitions in a variety of systems. Such models, however, being extensively studied in the frame of RG approach were actually treated only within the lowest-order (one- and two-loop) approximations which are known to lead to rather crude quantitative and, sometimes, to contradictory qualitative results.

The aim of this paper is to study the static critical behavior of the three-dimensional model with three quartic coupling constants on the base of three-loop RG series resummed in the way which provides proper physical predictions and accurate numerical estimates. As far as we know, this is the first attempt to get reliable, numerically correct results for a complicated 3D field-theoretical model from higher-order RG expansions [8]. The LandauWilson Hamiltonian of the model is as follows:

$$
\begin{aligned}
H=\frac{1}{2} \int d^{3} x & {\left[m_{0}^{2} \varphi_{\alpha} \varphi_{\alpha}^{*}+\nabla \varphi_{\alpha} \nabla \varphi_{\alpha}^{*}+\frac{u_{0}}{2} \varphi_{\alpha} \varphi_{\alpha}^{*} \varphi_{\beta} \varphi_{\beta}^{*}\right.} \\
+ & \left.\frac{v_{0}}{2} \varphi_{\alpha} \varphi_{\alpha} \varphi_{\alpha}^{*} \varphi_{\alpha}^{*}+\frac{w_{0}}{2} \varphi_{\alpha} \varphi_{\alpha} \varphi_{\beta}^{*} \varphi_{\beta}^{*}\right]
\end{aligned}
$$

where $\varphi_{\alpha}$ is a complex vector order parameter field, $\alpha, \beta=1,2, \ldots, N$, a bare mass squared $m_{0}^{2}$ being proportional to the deviation from the mean-field transition point (line).

This model describes critical phenomena in a plenty of substances. Their list includes 
tetragonal, hexagonal and cubic superconductors with $d$ - or $p$-wave pairing [9] as well as superconductors with two $-s$ and $d$ - order parameters [10], fully frustrated Josephsonjunction arrays (FFJJA) at zero temperature [11], stacked triangular antiferromagnets (STA) 112,13, helical magnets (HM) and magnets with sinusoidal spin structures [13] [16], the A phase of superfluid ${ }^{3} \mathrm{He}$ 17, 18]. The model Eq. (1.1) is related also to the critical thermodynamics of type-II superconductors with short coherence length near the upper critical magnetic field [19].

All the systems mentioned were extensively studied during the last decade and rich theoretical information about their critical behavior has been obtained both analytically and numerically. Unfortunately, the major part of these data turns out to be contradictory or inconclusive. To illustrate this point we overview, in brief, what was predicted for FFJJA, STA and HM by different people within different approaches.

A superconductor-insulator transition in FFJJA at zero temperature produced by competition of Josephson and charging effects in the presence of quantum fluctuations is described by three-dimensional model Eq.(1.1) with $N=2$ and $v_{0}=0$ or $w_{0}=0$. Starting from $4-\epsilon$ dimensions, such a transition was shown to be, within the lowest order in $\epsilon$, discontinuous [20], while $(2+\epsilon)$-expansion did not enable one to resolve whether it should be first-order or continuous [20]. On the other hand, this transition was referred to as second-order one on the base of analysis valid to the leading order in $1 / N$ [11].

For $N=2$ and $N=3$, the Hamiltonian (1.1) governs the critical behavior of STA such as $\mathrm{VCl}_{2}, \mathrm{VBr}_{2}, \mathrm{CsMnBr}_{3}, \mathrm{CsVCl}_{3}$, and of $\mathrm{HM}\left(\mathrm{Ho}, \mathrm{Dy}, \mathrm{Tb}, \beta-\mathrm{MnO}_{2}, \mathrm{MnAu}_{2}\right.$ ). In the case of Heisenberg spins, RG calculations in $4-\epsilon$ dimensions and $1 / N$-expansion result in a firstorder phase transition [14 16,21], although $\epsilon$-expansion predictions were believed also as favoring continuous one [13]. Monte-Carlo simulations in $3 D$ seem to provide an evidence of continuous phase transition [12,22]. RG analysis of corresponding $(2+\epsilon)$-dimensional model proposes that systems mentioned should undergo, in three dimensions, a first-order transition or a second-order one with either $O(4)$ (not $O(6)$ ) critical or tricritical mean-field exponents [23].

Obviously, the situation needs to be cleared up. Since the problem does not allow exact solution, in order to obtain reliable theoretical information one has to employ approximate methods with controlled or, at least, known level of accuracy. Regular RG perturbation 
theory in $3 D$ subject to the application of Padé-Borel-like resummation technique will be shown to play a role of such a method.

The paper is organized as follows. In Sec. [1] the Hamiltonians describing the systems mentioned above are considered and related to the Hamiltonian (1.1). In Sec. [II the renormalization scheme is formulated and three-loop RG expansions for $\beta$-functions and critical exponents are presented. Various resummation techniques based on the Borel transformation and applicable to divergent (asymptotic) power series of several independent variables are considered and criterions for the choise of the best one are established. The specific symmetries of the model (1.1) with $N=2$ are discussed in detail. They relate coordinates of different fixed points of $R G$ equations to each other being a sensitive indicator of the quality of the approximation employed. All the numerical results obtained are presented in Sec. 파 coordinates of the fixed points, critical exponent values, critical (marginal) order parameter dimensionalities $N_{c}$ at which the topology of flow diagrams changes, etc. RG flows are also shown in the planes where stable, within these planes, fixed points exist. In Sec. $\nabla$ the results obtained are applied to superconducting, superfluid and magnetically-order systems and certain theoretical predictions are made. Particular attention is paid to what is known as chiral critical behavior and its relevance to real HM and STA with Heisenberg or $X Y$-like spins. Sec. V] containes a summary of the results obtained. Details of Padé-Borel resummation procedure are described in Appendix.

\section{RELEVANT SUBSTANCES AND STRUCTURES}

In this section we discuss physical systems undergoing phase transitions which are described by the Hamiltonian (1.1).

\section{A. Unconventional superconductors}

These materials should be mentioned first since Eq. (1.1) is actually an obvious generalization of appropriate Ginzburg-Landau form (see, e.g. Ref. [9]) with $\varphi_{\alpha}$ being a superconducting order parameter. For $N=2$ the Hamiltonian under consideration governs a static

critical behavior of tetragonal and hexagonal $\left(v_{0}=0\right)$ superconductors with $d$-wave pairing, 
while the case $N=3$ corresponds to cubic $p$-wave materials. Heavy-fermion compounds such as $\mathrm{UPt}_{3}, \mathrm{UBe}_{13}$, and others are thought to belong to this class of superconductors 24,25]. Phase transitions in thorium-doped $\mathrm{UBe}_{13}$ are well described by the phenomenological model with two coexisting, $s-$ and $d$-wave, order parameters [10,26] which, in some limit, is reduced to that given by Eq. (1.1). Moreover, since there are numerous experimental facts [27,28] and theoretical predictions [29,30] favoring non-trivial pairing modes in high- $T_{c}$ superconductors, the Hamiltonian (1.1) may be also relevant to the critical behavior of these new materials [31].

It is worthy to note that the width of critical region is large enough in high- $T_{c}$ superconductors (see Refs. [32,33] for an overview and numerical estimates) and superconducting fluctuations proved to be clearly seen in their thermodynamics near $T_{c}$ [34 37. Wide fluctuation regions are also expected to exist in heavy-fermion compounds [38,39]. That is why the critical behavior of the model (1.1) is extensively studied within the context of superconductivity [40 42]. On the other hand, the Hamiltonian (11.1) has only one, isotropic gradient invariant, i.e. it ignores a crystallographic anisotropy of the order parameter correlation function which may play an appreciable role in the critical region. So, the applicability of the Eq. (1.1) to real unconventional superconductors is somewhat limited. The influence of anisotropic gradient terms on thermodynamics of these materials in the region of weak, Gaussian fluctuation was studied in Ref. [43].

\section{B. Fully frustrated Josephson-junction arrays}

Main features of JJA behavior are known to be described by the following Hamiltonian [44]:

$H=-\frac{E_{c}}{2} \sum_{i}\left(\frac{\partial}{\partial \theta_{i}}\right)^{2}-E_{J} \sum_{<i j>} \cos \left(\theta_{i}-\theta_{j}-A_{i j}\right)$,

where $\theta_{i}$ is a phase of superconducting order parameter in $i$-th island,

$$
A_{i j}=\frac{2 \pi}{\Phi_{0}} \int_{i}^{j} \mathbf{A} d \ell,
$$

A being a vector potential of external magnetic field, and $\Phi_{0}$ is a quantum of flux. Here $E_{c}$ plays a role of charging energy which is responsible for the Coulomb blockade and quantum 
dynamics while the Josephson coupling $E_{J}$ favors establishing of the global phase coherence and overall superconductivity in the system. At zero temperature superconductor-toinsulator transition occurs when the ratio $E_{c} / E_{J}$ exceeds a critical value. Since quantum fluctuations are essential in the case considered, the effective dimensionality of the system turns out to be equal to three: $D=2+1$ (see, e.g. Ref. [11]).

If the external magnetic field $\mathbf{B}$ is uniform JJA is regulary frustrated with the frustration parameter $f=\left(B a_{0}\right) / \Phi_{0}, a_{0}$ being an area of a plaquette. We shall consider JJA with square and triangular lattices in a magnetic field corresponding to $f=\frac{1}{2}$ which are usually referred to as fully frustrated ones. To study their critical behavior a proper Hubbard-Stratonovich transformation [11,45] may be applied to the model (2.1) resulting in the Landau-Wilson Hamiltonian with quartic terms which are, in notations of Ref. [11], as follows:

$u\left(\left|\psi_{1}\right|^{2}+\left|\psi_{2}\right|^{2}\right)^{2}-v_{1}\left|\psi_{1}\right|^{2}\left|\psi_{2}\right|^{2}+v_{2} \operatorname{Re}\left(\psi_{1}^{*} \psi_{2}\right)^{2}$,

where $\psi_{1}$ and $\psi_{2}$ are complex scalar fields. In the case of square lattice $u>0, v_{1}=v_{2}>0$, while for the triangular FFJJA $u>0, v_{1}<0$ and $v_{2}=0$. It is easy to see that Eq. (2.3) is actually identical to the quartic part of the Hamiltonian (1.1) for $N=2$ provided coupling constants are related to those standing in Eq. (1.1) by

$u=u_{0}+v_{0}+w_{0}, \quad v_{1}=2\left(v_{0}+w_{0}\right), \quad v_{2}=2 w_{0}$.

Domains $v_{0}=0, w_{0}>0$ and $v_{0}<0, w_{0}=0$ correspond to the square and triangular FFJJA respectively. The Hamiltonian (1.1) governs also the critical behavior of triangular JJA with $f=\frac{1}{4}$ since it is known to belong to the same universality class as FFJJA with square lattice 45].

\section{Stacked triangular antiferromagnets}

Triangular antiferromagnets which we shall deal with possess lattices consisting of triangular antiferromagnetic layers stacked in register along the orthogonal axis. In the ground state the spin arrangement may be thought as formed by three ferromagnetic sublattices with $120^{\circ}$ angles between neighboring, within the layer, spins (see Refs. [13,46] for detail). The microscopic Hamiltonian modelling STA reads [13]: 
$H=-J \sum_{<i j>} \mathbf{S}_{i} \mathbf{S}_{j}-J^{\prime} \sum_{<i j>} \mathbf{S}_{i} \mathbf{S}_{j}, \quad J<0$.

The first sum represents antiferromagnetic interactions within triangular layers which give rise to frustration. The second one describes interlayer coupling, the sign of $J^{\prime}$ being unimportant since there is no frustration along the orthogonal direction. The HubbardStratonovich transformation followed by the expanding around the instability points and other standart procedures leads to effective Hamiltonian containing

$$
u_{k}\left(\mathbf{a}^{2}+\mathbf{b}^{2}\right)+v_{k}\left[(\mathbf{a b})^{2}-\mathbf{a}^{2} \mathbf{b}^{2}\right]
$$

as an interaction term, a, b being real $n$-component vector fields 13 . If then one put

$$
u_{k}=u_{0}+w_{0}, \quad v_{k}=4 w_{0}
$$

Eq. (2.6) will immediately turn into the quartic part of the Hamiltonian (1.1) with $v_{0}=0$ and $\varphi_{\alpha}=a_{\alpha}+i b_{\alpha}$. The frustration may be shown to be relevant only for $w_{0}>0$; the opposite case, $w_{0}<0$, corresponds to simple ferromagnetic or antiferromagnetic ordering 46.

\section{Helical magnets}

In these magnets spins aling ferromagnetically in a plane and form spirals along the orthogonal axis. Such an ordering may be described by the microscopic Hamiltonian (2.5) provided the first and second sums are defined in a new manner [13]. Namely, let the first sum represents nearest-neighbor ferromagnetic interactions, $J>0$, while the second term in (2.5) describes antiferromagnetic, $J^{\prime}<0$, next-nearest-neighbor interactions acting along only one crystallographic axis. Then for ratios $\left|J^{\prime}\right| / J$ exceeding a critical value spins will be helically arranged along this axis. All the machinery mentioned above gives in this case just the same Landau-Wilson Hamiltonian as for STA. The helical ordering, however, is realized

only if $v_{k}>0\left(w_{0}>0\right)$ [13]. For $v_{k}<0$ a sinusoidal (linearly-polarized) spin density wave should occur [16]. 


\section{E. Superfluid helium-3}

In liquid ${ }^{3}$ He fermionic excitations are known to form, below $T_{c}$, Cooper-like pairs with $S=L=1$. Since magnetic dipole-dipole interaction couples orbital and spin angular momenta to each other the superfluid order parameter possesses a symmetry $O(3) \times U(1)$. This is precisely the symmetry underlying the Hamiltonian (1.1) with $N=3$ and $v_{0}=0$. As was shown in Refs. [17,:18, Eq. (1.1) describes, in fact, the transition of liquid helium-3 from normal to superfluid Anderson-Morel phase; coupling constants $g_{0}$ and $\lambda_{0}$ entering formulas of Refs. [17,18] are easily seen to be identical to $u_{0}$ and $w_{0}$ respectively.

\section{RG SERIES, RESUMMATION AND SYMMETRIES}

As was already mentioned, the static critical behavior of the model Eq. (1.1) has been studied in three dimensions within one- and two-loop RG approximations [42]. The taking into account of two-loop contributions to the $\beta$-functions and critical exponents was found to change drastically the results of the lowest-order RG analysis. In particular, it alters the total number of fixed points and avoids the degeneracy of the $O(2 N)$-symmetric fixed point which is four-fold degenerate, for $N=2$, within the parquette approximation. On the

other hand, some of the numerical results obtained on the base of the resummed two-loop RG expansions do not obey some exact symmetry relations (see below). In such a situation three-loop calculations turn out to be very desirable.

We calculate the $\beta$-functions for the Hamiltonian Eq. (1.1) within a massive theory. The renormalized Green function $G_{R}(p, m)$ and four-point vertex functions $U_{R}\left(p_{i}, m, u, v, w\right)$, $V_{R}\left(p_{i}, m, u, v, w\right), W_{R}\left(p_{i}, m, u, v, w\right)$ are normalized at zero momenta in a conventional way:

$$
\begin{gathered}
G_{R}^{-1}(0, m)=m^{2}, \\
\left.\frac{\partial G_{R}^{-1}(p, m)}{\partial p^{2}}\right|_{p^{2}=0}=1, \\
U_{R}(0, m, u, v, w)=m u, \\
V_{R}(0, m, u, v, w)=m v, \\
W_{R}(0, m, u, v, w)=m w .
\end{gathered}
$$

One extra condition is imposed on the $\varphi^{2}$ insertion: 


$$
\left.\Gamma_{R}^{(1,2)}(p, q, m, u, v, w)\right|_{p=q=0}=1
$$

The value of the one-loop vertex graph at zero external momenta including the factor $(N+4)$ is absorbed in $u, v, w$ in order to make the coefficient for $u^{2}$ term in $\beta_{u}$ equal to unity. The $\beta$-functions obtained are as follows:

$$
\begin{aligned}
& \beta_{u}=u-u^{2}-\frac{4}{N+4}\left(u v+u w+w^{2}\right)+\frac{2}{27(N+4)^{2}}\left[(41 N+95) u^{3}+200 u^{2} v+200 u^{2} w\right. \\
& \left.+46 u v^{2}+(46 N+216) u w^{2}+92 u v w+144 v w^{2}+(36 N+72) w^{3}\right] \\
& -\frac{1}{4(N+4)^{3}}\left[\left(2.69789 N^{2}+54.94038 N+99.82021\right) u^{4}+(26.58751 N\right. \\
& +329.22770)\left(u^{3} v+u^{3} w\right)+(2.48756 N+221.36225)\left(u^{2} v^{2}+2 u^{2} v w\right)+\left(2.48756 N^{2}\right. \\
& +155.55980 N+470.42246) u^{2} w^{2}+50.50080\left(u v^{3}+3 u v^{2} w\right)+(34.28057 N \\
& +626.66599) u v w^{2}+\left(8.11011 N^{2}+125.31213 N+311.16081\right) u w^{3}+110.42034 v^{2} w^{2} \\
& \left.+(1.95355 N+216.93358) v w^{3}+\left(-5.20190 N^{2}-0.62829 N+95.22334\right) w^{4}\right], \\
& \beta_{v}=v\left[1-\frac{2}{N+4}\left(3 u+\frac{5}{2} v+4 w\right)+\frac{2}{27(N+4)^{2}}\left[(23 N+185) u^{2}+362 u v+524 u w\right.\right. \\
& \left.+136 v^{2}+380 v w+(28 N+180) w^{2}\right]-\frac{1}{4(N+4)^{3}}\left[\left(-2.50221 N^{2}\right.\right. \\
& +41.85390 N+234.66699) u^{3}+(-0.01437 N+720.91540) u^{2} v+(8.98498 N \\
& +1015.38106) u^{2} w+579.33309 u v^{2}+1575.28532 u v w+(151.47423 N \\
& +780.92014) u w^{2}+157.45847 v^{3}+604.53412 v^{2} w+(6.49576 N+753.08966) v w^{2} \\
& \left.\left.+\left(-3.27046 N^{2}+13.63522 N+284.67391\right) w^{3}\right]\right] \text {, } \\
& \beta_{w}=w\left[1-\frac{2}{N+4}\left(3 u+v+\frac{N}{2} w\right)+\frac{2}{27(N+4)^{2}}\left[(23 N+185) u^{2}+200 u v+(54 N\right.\right. \\
& \left.+92) u w+28 v^{2}+56 v w+(36-8 N) w^{2}\right]-\frac{1}{4(N+4)^{3}}\left[\left(-2.50221 N^{2}+41.85390 N\right.\right. \\
& +234.66699) u^{3}+(-9.01372 N+426.44974) u^{2} v+\left(2.99978 N^{2}+83.14193 N\right. \\
& +230.13930) u^{2} w+162.71394 u v^{2}+(29.26715 N+266.89358) u v w+\left(5.75601 N^{2}\right. \\
& +48.11146 N+131.38337) u w^{2}+25.29977 v^{3}+(1.15422 N+73.59085) v^{2} w \\
& \left.\left.+(9.52258 N+106.38551) v w^{2}+\left(-1.31497 N^{2}+10.71074 N+58.66955\right) w^{3}\right]\right] .
\end{aligned}
$$


Such series are known to be divergent, at best asymptotic. They contain, however, rich and important physical information which may be extracted provided some procedure making them convergent is applied. The Borel transformation usually plays a role of this procedure. Here we are dealing with expansions of quantities depending on three variables $u, v$, and $w$. So, Borel transformation should be taken in the generalized form:

$f(u, v, w)=\sum_{i j k} c_{i j k} u^{i} v^{j} w^{k}=\int_{0}^{\infty} e^{-t} F(u t, v t, w t) d t$

where the Borel transform expansion is as follows:

$$
F(x, y, z)=\sum_{i j k} \frac{c_{i j k}}{(i+j+k) !} x^{i} y^{j} z^{k}
$$

To calculate the integral entering Eq. (3.4) one should perform an analytical continuation of the Borel-transformed expansion. Although there are several different ways to do it, only two approaches proved to be efficient in the case of multi-variable RG series [4.5]. The first one exploits the so called resolvent series 47]:

$\tilde{F}(x, y, z, \lambda)=\sum_{n=0}^{\infty} \lambda^{n} \sum_{l=0}^{n} \sum_{m=0}^{n-l} \frac{c_{l, m, n-l-m} x^{l} y^{m} z^{n-l-m}}{n !}$

which is actually a series in powers of $\lambda$ with coefficients $A_{n}$ being uniform polynomials of $n$-th order in $u, v$, and $w$. Padé approximants in $\lambda[L / M]$ is then used and the sum of the series is given by

$$
F(x, y, z)=\left.[L / M]\right|_{\lambda=1}
$$

(see Appendix for detail). This approximation scheme possesses the remarkable property: for $y=z=0$ ( or $x=z=0$, or $x=y=0$ ) expression (3.7) turns into conventional single-variable Padé approximants. Hence, all the results obtained for simpler, say, $O(n)-$ symmetric models hold good within this approach.

Another way of the analytic continuation is realized through the construction of the Canterbury approximants invented by Chisholm 48]:

$[K, L, M / R, P, Q]=\frac{\sum_{k=0}^{K} \sum_{l=0}^{L} \sum_{m=0}^{M} A_{k l m} x^{k} y^{l} z^{m}}{\sum_{r=0}^{R} \sum_{p=0}^{P} \sum_{q=0}^{Q} B_{r p q} x^{r} y^{p} z^{q}}$. 
It was found to be rather effective when applied to the impure Ising model [3, 4], the cubic model [4, 6], and the $m n-$ model [7].

To determine which approximation scheme is the most adequate to our problem certain criterious should be formulated. We adopt the following ones:

i) the resummation technique chosen should not lead to unphysical results,

ii) new results should be consistent with the most accurate numerical estimates for $O(n)-$ symmetric and other simple models known up today,

iii) new results should be self-consistent, i.e. numerical values of any critical exponent calculated by means of the resummation of different expansions, say, expansions for $\gamma$ and $\gamma^{-1}$, should be identical (as close as possible),

iv) all (known) symmetries of the problem should be preserved by the approximation scheme employed.

The last criterion is of prime importance in the case considered. The point is that the model Eq. (1.1) for $N=2$ possesses specific symmetry properties. Indeed, if the field $\varphi_{\alpha}$ undergoes the transformation

$$
\varphi_{1} \rightarrow \varphi_{1} \quad, \quad \varphi_{2} \rightarrow i \varphi_{2}
$$

quartic coupling constants are also transformed:

$$
u \rightarrow u \quad, \quad v \rightarrow v+2 w \quad, \quad w \rightarrow-w
$$

but the structure of the Hamiltonian itself remains unchanged [42]. Just the same situation takes place in the case of another field transformation [11,13]:

$$
\varphi_{1} \rightarrow \frac{\varphi_{1}+i \varphi_{2}}{\sqrt{2}} \quad, \quad \varphi_{2} \rightarrow \frac{i \varphi_{1}+\varphi_{2}}{\sqrt{2}}
$$

which does not affect the Hamiltonian structure resulting only in the following replacement of $u, v$, and $w$ :

$u \rightarrow u+v+2 w \quad, \quad v \rightarrow-2 w \quad, \quad w \rightarrow-\frac{v}{2}$

It is well known that RG functions of the problem are completely determined by the structure of the Hamiltonian: they do not depend on $u_{0}, v_{0}$, and $w_{0}$ which play a role of initial values of effective coupling constants when the $\mathrm{RG}$ flow of $u, v$, and $w$ is searched. 
Hence, RG equations should be invariant with respect to any transformation conserving the structure of the Hamiltonian 49]; Eqs. (3.10) and (3.12), in particular, were shown to be such transformations.

It means that for $N=2 \beta_{u}, \beta_{v}$, and $\beta_{w}$ should obey some special symmetry relations which may be readily written down:

$$
\begin{aligned}
& \beta_{u}(u, v, w)=\beta_{u}(u, v+2 w,-w), \\
& \beta_{v}(u, v, w)+2 \beta_{w}(u, v, w)=\beta_{v}(u, v+2 w,-w), \\
& \beta_{w}(u, v, w)=-\beta_{w}(u, v+2 w,-w) .
\end{aligned}
$$

and

$$
\begin{array}{r}
\beta_{u}(u, v, w)+\beta_{v}(u, v, w)+2 \beta_{w}(u, v, w)= \\
=\beta_{u}\left(u+v+2 w,-2 w,-\frac{v}{2}\right) \\
\beta_{v}(u, v, w)=-2 \beta_{w}\left(u+v+2 w,-2 w,-\frac{v}{2}\right) \\
2 \beta_{w}(u, v, w)=-\beta_{v}\left(u+v+2 w,-2 w,-\frac{v}{2}\right) .
\end{array}
$$

One can see that expansions Eqs. (3.3a, 3.3b, 3.30) do really satisfy these relations. Moreover, due to this special symmetry, transformations Eqs. (3.10) and (3.12) can, at most, rearrange the fixed points of RG equations not affecting numerical values of their coordinates $u_{c}, v_{c}$, and $w_{c}$ themselves. It provides powerful tool for evaluation of the accuracy of the approximation scheme employed.

To calculate critical exponents field-theoretical expansions for two of them are needed. We find $\gamma^{-1}$ and $\eta$ as power series in $u, v$, and $w$ up to three-loop order. They are as follows:

$$
\begin{aligned}
\gamma^{-1}= & -\frac{1}{N+4}\left[\frac{N+1}{2} u+v+w\right]+\frac{1}{(N+4)^{2}}\left[\frac{N+1}{2} u^{2}+2(u v+u w+v w)+v^{2}+N w^{2}\right] \\
& -\frac{0.2472701}{(N+4)^{3}}\left[\left(N^{2}+5 N+4\right) u^{3}+(6 N+24)\left(u^{2} v+u^{2} w+v w^{2}\right)+10\left(3 u v^{2}+6 u v w\right.\right. \\
& \left.\left.+v^{3}+3 v^{2} w\right)+(18 N+12) u w^{2}+\left(2 N^{2}+8\right) w^{3}\right]-\frac{0.1925093}{(N+4)^{3}}\left[\left(N^{2}+2 N+1\right) u^{3}\right. \\
& +\frac{18(N+1)}{3}\left(u^{2} v+u^{2} w\right)+(2 N+10)\left(u v^{2}+2 u v w\right)+\left(2 N^{2}+2 N+8\right) u w^{2} \\
& \left.+4\left(v^{3}+3 v^{2} w+N w^{3}\right)+(4 N+8) v w^{2}\right],
\end{aligned}
$$




$$
\begin{aligned}
\eta= & \frac{4}{27(N+4)^{2}}\left[(N+1) u^{2}+2\left(2 u v+2 u w+v^{2}+2 v w+N w^{2}\right)\right] \\
& +\frac{0.01234194}{(N+4)^{3}}\left[\left(N^{2}+5 N+4\right) u^{3}+(6 N+24)\left(u^{2} v+u^{2} w+v w^{2}\right)\right. \\
& \left.+10\left(3 u v^{2}+6 u v w+v^{3}+3 v^{2} w\right)+(18 N+12) u w^{2}+\left(2 N^{2}+8\right) w^{3}\right] .
\end{aligned}
$$

Since critical exponents are measurable (observable) quantities, the right-hand sides of Eqs. (3.15) and (3.16) should contain for $N=2$ only those combinations of $u, v$, and $w$ which are invariant under the transformations Eqs. (3.10) and (3.12). As may be seen, it is actually the case.

\section{NUMERICAL RESULTS}

So, we perform the resummation of three-loop expansions Eqs. (3.3a, 3.3b, 3.3a) by means of generalized Padé-Borel technique with the approximant [3/1] being used for the analytic continuation of Borel transforms. Coordinates of the fixed points of RG equations thus obtained are found numerically for the most interesting cases $N=2$ and $N=3$. They are presented in Table 【 $(N=2)$ and Table 【 $(N=3)$ which contain also, for comparison, the fixed point coordinates obtained earlier [42] from two-loop RG expansions resummed on the base of [2/1] Padé approximants. Three-loop contributions are seen to change appreciably locations of all non-trivial fixed points.

Let us first discuss the numerical accuracy of the values found. The point 2 in Tables $\mathbb{1}$ and $\mathbb{\Pi}$ is actually $O(2 N)$-symmetric fixed point and its coordinates are to be compared with those obtained for $O(4)$ - and $O(6)$-symmetric models with real fields $\varphi_{\alpha}$ from resummed highest-order RG series available up today. Four-loop calculations in $3 D$ have resulted in $u_{c}=1.377$ for $n=4$ and $u_{c}=1.338$ for $n=6$ [50]. These numbers differ from their

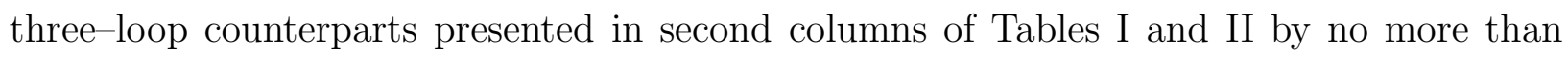
$1 \%$.

Third columns of the Tables [ and I] contain coordinates of the Bose $(X Y)$ fixed point. The most accurate estimate for $v_{c}$ obtained by the resummation of the six-loop 3D RG series is $v_{c}=1.405$ [1,2]. To compare this number with those presented in the Tables [ and $\mathbb{1}$, however, we should make some rescaling of $v_{c}$ for $N=2$ and $N=3$. The point is 
that the coefficient for $v^{2}$ in $\beta_{v}$ (Eq. (3.3b)) is equal to $\frac{5}{N+4}$ differing from unity for $N \neq 1$. Since the six-loop value of $v_{c}$ has been calculated in $O(2)$-symmetric model, i.e. for $N=1$, the numbers in third columns of Table [ I and II should be multiplyed, before comparison, by factors $\frac{5}{6}$ and $\frac{5}{7}$ respectively. It gives $v_{c}=1.4032(N=2)$ and $v_{c}=1.4033(N=3)$. Practical coincidence of these two values is very natural since they are actually coordinates of the same (Bose) fixed point while their closeness $(\sim 0.1 \%)$ to the six-loop value of $v_{c}$ provides an evidence of the high accuracy of the approximation scheme employed. Note, that the two-loop approximation leads to $v_{c}=1.5583$ which is more than $10 \%$ away from the "exact" value.

Strong evidences of the high numerical accuracy of the approach elaborated may be obtained on the base of symmetry arguments. As was shown above, transformations Eqs. (3.10) and (3.12) can only rearrange the fixed points of RG equations (3.3a, 3.3b, 3.3c) for $N=2$ not affecting the values of $u_{c}, v_{c}$, and $w_{c}$ themselves. Indeed, this is precisely what occurs when one applies Eq. (3.10) to the content of Table \&: the first four fixed points stay at their places while the points 5-8 undergo pair transpositions $5 \rightarrow 7,7 \rightarrow 5,6 \rightarrow 8,8 \rightarrow 6$. Another transformation, Eq. (3.12), practically does not change the location of fixed points 1, 2,7 , and 8 and causes pair transposition $3 \rightarrow 5,5 \rightarrow 3$. The rest of fixed points, the $4-$ th and the 6-th ones, however, are converted one to another under Eq. (3.12) only within three-loop approximation. Corresponding two-loop results turn out to violate the symmetry relations induced by Eq. (3.12). More precisely, the differences between the coordinates of the point 4 and the transformed coordinates of the point 6 ("symmetry discrepancies") given by [2/1] Padé-Borel approximants are about 0.3, while within three-loop approximation they are of order of 0.01 .

So, the three-loop terms being taken into account enable one to obtain results which are much more accurate than those given by two-loop RG expansions. Moreover, it is seen that the field-theoretical RG approach in three dimensions combined with a generalized Padé-Borel resummation technique does really provide a regular, rapidly converging approximation scheme powerful enough to treat complicated model with three quartic coupling constants. At the same time, the Chisholm-Borel resummation procedure is found to give poor results in this case.

Let us discuss further the stability of the fixed points and the structure of the RG 
flow diagrams. All fixed points of the RG equations are unstable in the three-dimensional parameter space $(u, v, w)$. The 4 -th and the 6-th ones, however, are stable within the planes $(u, v)$ and $(u, w)$ respectively. The existence of such points is important since it implies the possibility of continuous phase transitions in numerous physical systems described by the model Eq. (1.1) with $N=2$ and $v_{0}=0$ or $w_{0}=0$. RG flows for $N=2$ within the planes $(u, v)$ and $(u, w)$ and for $N=3$ within the plane $(u, v)$ are shown in Fig. 1)(b) and Fig. 2(b, c). One can see from these figures that there is not a fixed point stable within the plane $(u, w)$ for $N=3$ while for $N=2$ such a point exists. Hence, the topology of the flow diagram should change when $N$ varies. It is interesting, therefore, to study the structure of our RG flows for arbitrary $N$.

The detailed numerical analysis of three-loop RG equations obtained shows that only two diverse $u-v$ flow pictures occur for $1<N<\infty$ while the RG flow within the plane $(u, w)$ may proceed in four different ways. All possible scenarios are depicted in Fig. 1 and Fig. 2 (see hardcopy: Phys. Rev. B 49, 15901 (1994)). The critical (marginal) dimensionality of the order parameter $N_{c}$ which separates from each other two regimes of RG flows for $w=0$ is found to be:

$$
N_{c}=1.47 \pm 0.01
$$

Since this number is less than two, in all physically interesting cases, i.e. for $N \geq 2$, the $O(2 N)$-symmetric fixed point turns out to be unstable. So, the system should undergo either a continuous phase transition demonstrating an anisotropic $\left(v_{c} \neq 0\right)$ critical behavior or a fluctuation induced first-order phase transition. When $N \rightarrow \infty$ the anisotropic stable fixed point in the plane $(u, v)$ is going to the $O(2)$-symmetric one which becomes degenerate in this spherical-model limit.

The behavior of our model in the plane $(u, w)$ is more rich. It is characterized by three marginal values of the order parameter dimensionality: $N_{c 1}, N_{c 2}$, and $N_{c 3}$. Calculated on the base of resummed three-loop RG series Eqs. (3.3a, 3.3b, 3.30) they are as follows:

$$
\begin{aligned}
& N_{c 1}=1.45 \pm 0.01, \\
& N_{c 2}=2.03 \pm 0.01 \\
& N_{c 3}=3.91 \pm 0.01
\end{aligned}
$$


For $N<N_{c 1}$ (Fig. 2(a)) three non-trivial fixed points exist in the plane $(u, w)$ with the $O(2 N)$-symmetric point being stable. When $N$ exceeds $N_{c 1}$ this "Heisenberg" fixed point loses its stability but the other, anisotropic fixed point with $w_{c}<0$ acquires it (Fig. 2(b)). In this domain which includes the important case $N=2$, our system demonstrates an anisotropic scaling behavior or discontinuous phase transitions. With increasing $N$ the stable fixed point in Fig. (2) (b) is moving downward and "annihilates" with the anisotropic saddle fixed point when $N$ approaches $N_{c 2}$. There is only one non-trivial fixed point in the domain $N_{c 2}<N<N_{c 3}$ including $N=3$ (Fig. 2(c)); it is $O(2 N)$-symmetric and unstable. So, only first-order phase transitions are possible, in principle, in this case provided $w_{0} \neq 0$. At last, when $N$ increases further and crosses over the value $N_{c 3}$ the creation of two new anisotropic fixed points in the $u-w$ flow diagram takes place (Fig. 2(d)). One of them is stable and describes some anisotropic critical behavior with $w_{c}>0$. This fixed point is known as a "chiral" point 13 and corresponding "chiral" phase transition has been extensively studied during the last years. As follows from our estimates (Eq. (4.2)), this point does really exist and governs the scaling behavior of physical systems with $N \geq 4$. For $N=2$ and $N=3$ the chiral critical behavior does not actually realized.

Let's discuss numerical estimates Eqs. (4.1) and (4.2) in more detail. The value of $N_{c 2}$ turns out to be very close to $N=2$ which is of prime physical importance. Can higher-order contributions to the $\beta$-functions being taken into account change $N_{c 2}$, invert the inequality $N_{c 2}>2$, and, hence, alter the structure of the $u-w$ flow diagram for $N=2$ ? No, they can not. The point is that the structures of the RG flows in the planes $(u, v)$ and $(u, w)$ are related to each other for $N=2$ by the symmetry relations discussed earlier. In particular, as may be seen from Eq. (3.12) the total number of fixed points in each of these flow diagrams should be just the same. Since the plane $(u, v)$ definitely contains four fixed points $\left(N_{c}\right.$ lies far below the value of interest $N=2)$ the plane $(u, w)$ for $N=2$ should possess four fixed points too. Moreover, since, for $N=2$, the stable fixed point has $v_{c}>0$ its counterpart in the plane $(u, w)$ should possess $w_{c}<0$ (see Eq. (3.12) ). It means that inevitably $N_{c 2}>2$ in the exact theory. The estimate Eq. (4.2) is in accord with this inequality.

Another point to be discussed is the near coincidence of the calculated values of $N_{c}$ and $N_{c 1}$. It is not occasional. Indeed, $N_{c}$ and $N_{c 1}$ are both the values of $N$ for which the $O(2 N)$-symmetric fixed point becomes degenerate and critical exponents describing its 
stability change a sign. These exponents are completely determined by derivatives $\frac{\partial \beta_{v}}{\partial v}$ and $\frac{\partial \beta_{w}}{\partial w}$ taken at the "Heisenberg" fixed point since $\frac{\partial \beta_{v}}{\partial w}$ and $\frac{\partial \beta_{w}}{\partial v}$ at this point vanish. One can see from Eqs. (3.3b) and (3.3d), however, that $\frac{\partial \beta_{v}}{\partial v}=\frac{\partial \beta_{w}}{\partial w}$ along the whole line $v=w=0$ up to the highest calculated order. So, when $N$ varies the "Heisenberg" fixed point should lose its stability in planes $(u, v)$ and $(u, w)$ simultaneously, i.e. $N_{c}$ and $N_{c 1}$ should be equal to each other. The small difference between calculated values of $N_{c}$ and $N_{c 1}$ reflects a finite accuracy of our approximation scheme which is seen to be of order of 0.01 .

Having calculated fixed point coordinates we can find critical exponents for our model. To obtain accurate estimates for $\gamma$ the expansion Eq. (3.15) is resummed by means of the generalized Padé-Borel procedure described above while the values of $\eta$ are found by direct substitution of fixed point coordinates into Eq. (3.16) since this very short series with very small and positive three-loop term doesn't need in resummation. The results obtained for $N=2$ and $N=3$ are presented in Table $\mathbb{\Pi 1}$ and Table $\mathbb{\text { IV }}$ respectively which contain also the values of $\gamma$ and $\eta$ calculated earlier 42] within two-loop approximation.

Three-loop contributions are seen to change critical exponents values only slightly. For $N=2$ critical exponents calculated in the fixed points 3,5 , and 7 turn out to be almost identical, and so is true for the fixed points 4,6 , and 8 . In the exact theory each of these two sets of fixed points indeed should possess identical critical exponents since fixed points belonging to the same set are related to each other by symmetry relations Eqs. (3.10) and (3.12), i.e. they are actually the same fixed point. So, differences between the values of $\gamma$ and $\eta$ calculated in such fixed points may be considered as a measure of numerical accuracy of our approximation. It is seen to be better than 0.001. On the other hand, the difference between the values of $\gamma$ calculated in the fixed points 4 and 6 (or 8) within two-loop approximation exceeds 0.05. Hence, the taking into account of three-loop terms improves the situation essentially.

It is worthy also to compare critical exponents found in the Bose and "Heisenberg" fixed points with their counterparts determined from six-loop [1,2] and four-loop [50] RG expansions for a simple $O(n)$-symmetric model. The most accurate estimate for the susceptibility exponent of the $3 D X Y$ model is $\gamma=1.315$ [1,2]. Corresponding values in Tables [II and IV (third columns) differ from it by 0.005 . Four-loop RG calculations for $n=2 N=4$ and 
$n=2 N=6$ give $\gamma=1.441$ and $\gamma=1.541$ respectively [50]. Differences between these numbers and their three-loop twins presented in Tables III and IV (second columns) are about 0.02. So, we arrive to the conclusion: Padé-Borel resummed $3 D$ three-loop RG expansions provide an accuracy of order of 0.01 for all calculated quantities. This accuracy is sufficient for making definite and reliable theoretical prediction for physical systems described by the model Eq. (1.1). It will be done in the following Section.

Now let's return back to the calculation of critical exponents. The rest of them may be found by making use of well-known scaling relations. We present here numerical values of exponents $\nu, \alpha$, and $\beta$ for the fixed points which are stable within corresponding parameter subspaces since only these numbers may be related to experiments. So, for equivalent fixed points 4, 6, and 8 from Table 【II

$$
\nu=0.677, \quad \alpha=-0.030, \quad \beta=0.347
$$

while the point 4 in Table $\mathbb{\mathrm { IV }}$ is characterized by $\nu=0.673, \alpha=-0.020$, and $\beta=0.345$.

\section{APPLICATION TO PHYSICAL SYSTEMS AND DISCUSSION}

All fixed points of our RG equations were found to be unstable within the threedimensional parameter space $(u, v, w)$ for $N=2$ and $N=3$. It means that only discontinuous, first-order phase transitions should occur in physical systems with non-zero initial values of $v$ and $w$. Such systems are represented by cubic and tetragonal unconventional superconductors and superconductors with composite $s-d$ order parameters. On the other hand, fluctuation-driven first-order phase transitions are known to be extremely weak. So, the absence, within the experimental accuracy, of discontinuous superconducting transitions in relevant heavy-fermion and high- $T_{c}$ compounds does not actually contradict to the above conclusion.

In hexagonal $d$-wave superconductors described by the model Eq. (1.1) with $N=2$ and $v_{0}=0$ second-order phase transitions remain possible under strong superconducting fluctuations since there is a stable fixed point within the plane $(u, w)$ which possesses a sizable domain of attraction. Corresponding values of critical exponents (column $4(6,8)$ in Table [II and Eq. (4.3)) turn out to be close enough to those of the $3 D X Y$ model. 
So, it is actually impossible to distinguish between the BCS $s$-wave pairing and the nontrivial one studying experimentally the scaling behavior of superconductors. On the other hand, anisotropic gradient terms omitted in the Hamiltonian (1.1) can themselves change, in course of fluctuation renormalization, the order of phase transition and the structure of phase diagram, as they do in crystals undergoing structural (ferroelectric) and magnetic phase transitions [51,52]. This will obviously result in a non-universal behavior of hexagonal $d$-wave superconductors in the critical region.

In liquid helium-3, where $N=3$ and $v_{0}=0$ and, therefore, RG equations have no stable fixed points, fluctuations should always force the superfluid phase transition to be firstorder. Corresponding discontinuities of thermodynamic quantities at the transition point, however, would hardly be observed experimentally because of the narrowness of the critical region in this Fermi-liquid (see, e.g. Refs. [17,53] for numerical estimates).

Only first-order phase transitions should emerge also in FFJJA at $T=0$, in spite of the existence of stable fixed points in planes $(u, v)$ and $(u, w)$ for $N=2$. Indeed, RG trajectories starting from physical initial points, i.e. from those having $v_{0}<0$ and $w_{0}>0$ for triangular and square FFJJA respectively [11,45], can not achieve the stable fixed points as is cleary seen from Fig. 1(b) and Fig. 2(b). So, these systems will demonstrate non-universal critical behavior.

A mode of the critical behavior of STA and HM described by the Hamiltonian (1.1) with $v_{0}=0$ depends on the dimensionality of the order parameter. In materials with Heisenberg spins, i.e. for $N=3$, only (weak) first-order phase transitions should occur. In easy-plane crystals with $X Y$-like spins continuous transitions are also possible with critical exponents presented in column $4(6,8)$ of Table $\amalg I I$ and Eq. (4.3) which are practically undistinquishable from those of $3 D X Y$ model. These exponents, however, govern transitions into somewhat trivial phases: simple ferromagnetic or antiferromagnetic in STA and a sinusoidal (linearlypolarized) in HM, since relevant stable fixed point possesses $w_{c}<0$. Much more interesting ordering with frustration in STA and helical one in HM are described by Eq. (1.1) with $w_{0}>0$. They may be realized only via first-order phase transitions, as is clearly seen from Fig. 2(b).

We did not find any traces of chiral second-order phase transitions and corresponding new classes of universality for $N=2$ and $N=3$, i.e. for STA and HM with Heisenberg or 
$X Y$-like spins. This result is in contradiction with conjectures and conclusions made on the base of the lower-order $\epsilon$-expansion analysis [13, 16]. Such conclusions, however, can not be referred to as reliable since the method mentioned provides rather low numerical accuracy in three dimensions. To illustrate this point and to clear up the situation let's discuss two-loop $\epsilon$-expansions (highest-order now available) for marginal order-parameter dimensionalities $N_{c 1}, N_{c 2}$, and $N_{c 3}$. They are as follows [13]:

$$
\begin{aligned}
& N_{c 1}=2-\epsilon, \\
& N_{c 2}=2.20-0.57 \epsilon, \\
& N_{c 3}=21.8-23.4 \epsilon .
\end{aligned}
$$

When $\epsilon \rightarrow 1 N_{c 3}$ becomes less than 2 and chiral fixed point seems to exist for $N=2$ and $N=3$. In this limit, however, $N_{c 2}$ becomes less than 2 too what is in obvious contradiction with an inequality $N_{c 2}>2$ proven above. Moreover, another inequality $N_{c 2}<N_{c 3}$ valid for $\epsilon \ll 1$ turns out to be broken at $\epsilon=1$ as well.

Is it possible to make $\epsilon$-expansion predictions more accurate for $\epsilon=1$ ? Yes, of course. Higher-order (four- and five-loop) $\epsilon$-expansions are known to give rather good numerical results for $3 D O(n)$-symmetric model at $\epsilon=1$ provided some Borel-like resummation procedure is applied [54 56]. Unfortunately, we have no long enough $\epsilon$-expansions for our model up today [57]. So, we try to "sum up" expansions (5.19, 5.1b, 5.10) constructing simple Padé approximants:

$$
\begin{aligned}
& N_{c 1} \approx \frac{2}{1+0.5 \epsilon}, \\
& N_{c 2} \approx \frac{2.20}{1+0.26 \epsilon}, \\
& N_{c 3} \approx \frac{21.8}{1+1.07 \epsilon} .
\end{aligned}
$$

For $\epsilon=1$ these formulas give $N_{c 1}=1.33, N_{c 2}=1.75$, and $N_{c 3}=10.5$. The first number is much closer to our estimate $N_{c 1}=1.45$ (Eq. (4.2) ) than the value $N_{c 1}=1$ given by 
Eq. (5.19). The second one is also closer to the three-loop $3 D$ estimate $N_{c 2}=2.03$ than the naive value $N_{c 2}=1.63$, but both violate the inequality $N_{c 2}>2$. The third number exceeds enormously the estimate $N_{c 3}=3.91$ which turns out to lie between this number and the naive estimate $N_{c 3}=-1.6$. So, we see that primitive resummation of very short expansions (5.1a, 5.1b, 5.1d) results in somewhat improved numerical estimates for $N_{c 1}$ and $N_{c 2}$ while being used for evaluation of $N_{c 3}$ it demonstrates that lower-order $\epsilon$-expansions are useless in this case. Hence, lower-order calculations in $4-\epsilon$ dimensions can not be considered as a tool for answering the question whether $3 D$ physical systems with $N=2$ and $N=3$ undergo chiral phase transitions or not.

Monte-Carlo simulations [12,22] would also hardly be referred to as evidence of chiral critical behavior of STA and HM with Heisenberg or $X Y$-like spins. The point is that unusual values of critical exponents given by such calculations turn out to be close to tricritical ones. That's why it was suggested [23] that tricritical behavior or tricritical-to-critical crossover are really seen in these computer experiments as well as in most of physical experiments performed on several helimagnets (Tb, Dy, Ho) and $\mathrm{STA}\left(\mathrm{CsMnBr}_{3}, \mathrm{CsVCl}_{3}\right.$ and others). We completely agree with what is argued on this topic in Ref. 223] where the reader can find also an overview and analysis of relevant experimental data.

\section{CONCLUSIONS}

The critical behavior of the model describing phase transitions in superconducting and magnetic systems with complex $N$-vector order parameter as well as in superfluid helium3 has been studied within the RG approach in three dimensions. RG $\beta$-functions and critical exponents have been calculated as series in powers of renormalized quartic coupling constants $u, v$, and $w$ up to three-loop order. The series obtained have been resummed by means of the generalized Padé-Borel technique and fixed points coordinates, critical exponents values and a structure of RG flows have been determined for $N=2$ and $N=3$. Marginal values of the order parameter dimensionality at which the topology of RG flows in planes $(u, v)$ and $(u, w)$ changes have been also found. Several criterions have been used to estimate the accuracy of numerical results obtained which had turned out to be about 0.01, an order of magnitude better than that given by resummed two-loop RG expansions. 
So, field-theoretical RG approach in three dimensions combined with a proper resummation technique provides a regular, rapidly converging approximation scheme powerful enough to treat complicated model with three quartic coupling constants.

Relevant physical systems have been shown to undergo, in most cases, fluctuation induced first-order phase transitions. Second-order transitions have been found to occur only in hexagonal $d$-wave superconductors and in planar magnets (into somewhat trivial phases: linearly-polarized or unfrustrated). Corresponding critical exponents have turned out to differ from those of the $3 D X Y$ model by no more than $0.02-0.03$, i.e. the underlying critical behavior would hardly be thought as experimentally distinguishable from the Bose one. RG equations obtained have been shown to possess the chiral fixed point but only for $N \geq 4$. It means that STA and HM with Heisenberg and $X Y$-like spins would not really demonstrate the chiral critical behavior with unusual critical exponents approaching helical or frustrated antiferromagnetic states via first-order phase transitions.

\section{ACKNOWLEDGMENTS}

We gratefully acknowledge useful discussions with B. N. Shalayev. One of us (S. A. A.) would also thank D. A. Zuev for providing a considerable amount of computer time. This work was supported in part by the Scientific Council on the Problem of High Temperature Superconductivity via the Russian National Program "High Temperature Superconductivity" under Project No. 90476 "Gran", and in part by the Science and Higher School State Committee of Russian Federation through Grant No. 93-7.1-51.

In this Appendix, some details of the resummation procedure employed are described. As was shown in Sec. [II, resolvent series

$$
\begin{aligned}
& \tilde{F}(x, y, z ; \lambda)=\sum_{n=0}^{\infty} A_{n} \lambda^{n} \\
& A_{n}=\sum_{l=0}^{n} \sum_{m=0}^{n-l} \frac{c_{l, m, n-l-m}}{n !} x^{l} y^{m} z^{n-l-m}
\end{aligned}
$$


for Borel transforms of the original multi-variable RG expansions may be constructed to generate Padé approximants $[L / M]$ in parameter $\lambda$. These approximants are defined in a conventional way:

$$
[L / M]=\frac{P_{L}(\lambda)}{Q_{M}(\lambda)}
$$

where $P_{L}(\lambda)$ and $Q_{M}(\lambda)$ are polynomials of degrees $L$ and $M$ respectively with coefficients depending on $x, y$, and $z$, which may be determined from the following relations:

$$
\begin{aligned}
& Q_{M}(\lambda) \tilde{F}(x, y, z ; \lambda)-P_{L}(\lambda)=O\left(\lambda^{L+M+1}\right), \\
& Q_{M}(0)=1 .
\end{aligned}
$$

Approximate expressions for $\beta$-functions and critical exponents are then obtained by the replacement of variables $x=u t, y=v t$, and $z=w t$ in Padé approximants and by evaluation of the integral

$$
\left.\int_{0}^{\infty} e^{-t}[L / M]\right|_{\lambda=1} d t
$$

(Borel transformation).

With three-loop expansions in hand, we can use two different approximants [3/1] and [2/2] obeying the condition $L \geq M$. The former was shown (Sec. IV) to provide rather good numerical results for all cases considered. Moreover, an employment of this approximant kept us away from the well-known problem of poles which often arises when approximants with higher-order denominators are used. That's why we have chosen Padé approximant [3/1] for our analysis. When expressed in terms of renormalized coupling constants $u, v$, and $w$ and the variable $t$ it is as follows:

$$
[3 / 1]=\frac{a_{0}+a_{1} t+a_{2} t^{2}+a_{3} t^{3}}{1+b_{1} t},
$$

where $a_{0}, \ldots, a_{3}$ and $b_{1}$ are known functions of $u, v$, and $w$. If series to be resummed are those for $\beta$-functions the coefficient $a_{0}$ in Eq. (5) turns out to vanish and the integral (田) reads

$$
\int_{0}^{\infty} t e^{-t} \frac{a_{1}+a_{2} t+a_{3} t^{2}}{1+b_{1} t} d t
$$


Evaluating this integral we get the final expression (the "sum" of the series) for the function of interest:

$$
\begin{aligned}
f(u, v, w)= & \left(a_{1}+a_{2}+2 a_{3}\right) b-\left(a_{2}+a_{3}-a_{3} b\right) b^{2} \\
& +\left(a_{1}-a_{2} b+a_{3} b^{2}\right) b^{2} e^{b} E i(-b),
\end{aligned}
$$

where $\operatorname{Ei}(x)$ is the exponential integral [58] and $b=b_{1}^{-1}$. This is precisely the formula which was used for resummation of the three-loop RG expansions Eqs. (3.3a, 3.3b, 3.3a) and for determination of the fixed points. The approximate expression for $\gamma^{-1}(u, v, w)$ is quite similar and not presented here. 


\section{REFERENCES}

[1] G. A. Baker, Jr., B. G. Nickel, and D. I. Meiron, Phys. Rev. B 17, 1365 (1978).

[2] J. C. Le Guillou and J. Zinn-Justin, Phys. Rev. B 21, 3976 (1980).

[3] I. O. Maier and A. I. Sokolov, Fiz. Tverd. Tela (Leningrad) 26, 3454 (1984) [Sov. Phys. Solid State 26, 2076 (1984)].

[4] I. O. Mayer, A. I. Sokolov, and B. N. Shalayev, Ferroelectrics 95, 93 (1989).

[5] I. O. Mayer, J. Phys. A 22, 2815 (1989).

[6] I. O. Maier and A. I. Sokolov, Ferroelectric Lett. 9, 95 (1988).

[7] N. A. Shpot, Phys. Lett. A 142, 474 (1989).

[8] Some of the results obtained have been reported at the International Conference "Renormalization Group 91" (Dubna, USSR, 1991) and published in the Conference Proceedings: S. A. Antonenko and A. I. Sokolov, in Renormalization Group '91, edited by D. V. Shirkov \& V. B. Priezzhev (World Scientific, Singapore-New Jersey-LondonHong Kong, 1992), p.153.

[9] G. E. Volovik and L. P. Gor'kov, Zh. Eksp. Teor. Fiz. 88, 1412 (1985) [Sov. Phys. JETP 61, $843(1985)]$.

[10] P. Kumar and P. Wolfle, Phys. Rev. Lett. 59, 1954 (1987).

[11] E. Granato and J. M. Kosterlitz, Phys. Rev. Lett. 65, 1267 (1990).

[12] H. Kawamura, J. Phys. Soc. Jpn. 55, 2095 (1986); J. Appl. Phys. 63, 3086 (1988).

[13] H. Kawamura, Phys. Rev. B 38, 4916 (1988).

[14] S. A. Brazovskii, I. E. Dzyaloshinskii, and B. G. Kukharenko, Zh. Eksp. Teor. Fiz. 70, 2257 (1976).

[15] P. Bak and D. Mukamel, Phys. Rev. B 13, 5086 (1976).

[16] H. Kawamura, Phys. Rev. B 38, 960 (1988). 
[17] D. R. T. Jones, A. Love, and M. A. Moore, J. Phys. C 9, 743 (1976).

[18] D. Bailin, A. Love, and M. A. Moore, J. Phys. C 10, 1159 (1977).

[19] S. A. Ktitorov, Yu. V. Petrov, B. N. Shalayev, and V. S. Sherstinov, Fiz. Tverd. Tela (Leningrad) 33, 575 (1991); Int. J. Mod. Phys. B 6, 1209 (1992).

[20] M. Yosefin and E. Domany, Phys. Rev. B 32, 1778 (1985).

[21] T. Garel and P. Pfeuty, J. Phys. C 9, L245 (1976).

[22] H. T. Diep, Phys. Rev. B 39, 397 (1989).

[23] P. Azaria, B. Delamotte, and T. Jolicoeur, Phys. Rev. Lett. 64, 3175 (1990).

[24] D. E. MacLaughlin, C. Tien, W. G. Clark, M. D. Lan, Z. Fisk,J. L. Smith, and H. R. Ott, Phys. Rev. Lett. 53, 1833 (1984).

[25] W. K. Kwok, L. E. DeLong, G. W. Crabtree, and D. G. Hinks, Phys. Rev. B 41, 11649 (1990).

[26] A. Langner, D. Sahu, and T. F. George, Phys. Rev. B 38, 9187 (1988).

[27] K. Ishida, Y. Kitaoka, T. Yoshitomi, N. Ogata, T. Kamino, and K. Asayama, Physica C 179, 29 (1991).

[28] Q. P. Li and R. Joint, Phys. Rev. B 47, 530 (1993).

[29] G. Kotliar, Phys. Rev. B 37, 3664 (1988).

[30] P. Monthoux, A. V. Balatsky, and D. Pines, Phys. Rev. B 46, 14803 (1992).

[31] We mean here high-temperature superconductors with moderate anisotropy such as $\mathrm{YBa}_{2} \mathrm{Cu}_{3} \mathrm{O}_{7}$ which demonstrate well-pronounced $3 \mathrm{D}$ behavior in the vicinity of $\mathrm{T}_{c}$.

[32] A. I. Sokolov, Physica C 174, 208 (1991).

[33] A. I. Sokolov, Sverkhprovodimost': Fizika, Khimiya, Tekhnika 5, 1794 (1992).

[34] S. E. Inderhees, M. B. Salamon, J. P. Rice, and D. M. Ginzberg, Phys. Rev. Lett. 66, $232(1991)$. 
[35] E. Braun, W. Schnelle, H. Broicher, J. Harnischmacher, D. Wohlleben, C. Allgeier, W. Reith, J. S. Schilling, J. Bock, E. Preisler, and G. J. Vogt, Z. Phys. B 84, 333 (1991).

[36] W. C. Lee, J.-H. Cho, and D. C. Johnston, Phys. Rev. B 43, 457 (1991).

[37] A. S. Panfilov, A. I. Smirnov, and L. B. Kuzmicheva, Fizika Nizkikh Temperatur 17, 1152 (1991).

[38] V. V. Moshchalkov, K. Svozil, Phys. Lett. A 120, 356 (1987).

[39] J. P. Brison, J. Flouquet, and G. Deutscher, J. Low Temp. Phys. 76, 453 (1989).

[40] Yu. M. Ivanchenko, A. A. Lisyanskii, and A. E. Filippov, Fiz. Tverd. Tela (Leningrad) 31, 204 (1989).

[41] E. J. Blagoeva, G. Busiello, L. De Cesare, Y. T. Millev, I. Rabuffo, and D. I. Uzunov, Phys. Rev. B 40, 7357 (1989); Phys. Rev. B 42, 6124 (1990).

[42] S. A. Antonenko, A. I. Sokolov, and B. N. Shalaev, Fiz. Tverd. Tela (Leningrad) 33, 1447 (1991) [Sov. Phys. Solid State 33, 815 (1991)].

[43] J. F. Annett, M. Randeria, and S. R. Renn, Phys. Rev. B 38, 4660 (1988).

[44] R. M. Bradley and S. Doniach, Phys. Rev. B 30, 1138 (1984).

[45] M. Y. Choi and S. Doniach, Phys. Rev. B 31, 4516 (1985).

[46] H. Kawamura, J. Phys. Soc. Jpn. 55, 2157 (1986).

[47] G. A. Baker, Jr. and P. Graves-Morris. Padé Approximants (Addison-Wesley, Reading, MA, 1981).

[48] J. S. R. Chisholm, Math. Comp. 27, 841 (1973).

[49] For a simple anisotropic model with two-component real order parameter it was shown by A. L. Korzhenevskii, Zh. Eksp. Teor. Fiz. 71, 1434 (1976).

[50] I. O. Maier, A. I. Sokolov, Abstracts of the Seventeenth All-Union Conference on the Physics of Magnetic Phenomena (Donetsk, USSR, 1985), pp.26-27; I. O. Maier, 
Ph. D. Thesis, A. F. Ioffe Physico-Technical Institute, Leningrad, (1990), unpublished.

[51] T. Nattermann and S. Trimper, J. Phys. A8, 2000 (1975).

[52] A. I. Sokolov, A. K. Tagantsev, Zh. Eksp. Teor. Fiz. 76, 181 (1979) [Sov. Phys. JETP 49, $92(1979)]$.

[53] A. I. Sokolov, Zh. Eksp. Teor. Fiz. 78, 1985 (1980) [Sov. Phys. JETP 51, 998 (1980)].

[54] A. A. Vladimirov, D. I. Kazakov, O. V. Tarasov, Zh. Eksp. Teor. Fiz. 77, 1035 (1979).

[55] S. G. Gorishny, S. A. Larin, F. V. Tkachov, Phys. Lett. 101A, 120 (1984).

[56] J. C. Le Guillou, J. Zinn-Justin, J. Physique Lett. 46, L137 (1985).

[57] Three-loop calculations for the model Eq. (1.1) in $4-\epsilon$ dimensions are now in progress.

[58] The following definition of $E i(x)$ is adopted here: $E i(x)=\int_{-\infty}^{x} e^{t} t^{-1} d t$. 


\section{FIGURES}

FIG. 1. RG flows in the plane $(u, v)$ for $N<N_{c}$ and $N>N_{c}$ where $N_{c}=1.47 \pm 0.01$. Shaded areas represent the regions of instability of the Hamiltonian (1.1) (see hardcopy: Phys. Rev. B 49, $15901(1994))$.

FIG. 2. Four possible scenarios of RG flow in the plane $(u, w)$. Marginal values of the order

parameter dimensionality $N_{c 1}, N_{c 2}$, and $N_{c 3}$ are given by Eq. (4.2). Shaded areas are the regions of instability of the Hamiltonian (1.1) (see hardcopy: Phys. Rev. B 49, 15901 (1994)). 


\section{TABLES}

TABLE I. Coordinates of the fixed points of $\mathrm{RG}$ equations for $N=2$ obtained within three-loop (approximant [3/1]) and two-loop (approximant [2/1]) approximations.

\begin{tabular}{|c|c|c|c|c|c|c|c|c|c|}
\hline & & 1 & 2 & 3 & 4 & 5 & 6 & 7 & 8 \\
\hline \multirow[t]{2}{*}{$u_{c}$} & {$[3 / 1]$} & 0.0 & 1.3671 & 0.0 & 0.1872 & 1.6833 & 1.6787 & 1.6832 & 1.6789 \\
\hline & {$[2 / 1]^{*}$} & 0.0 & 1.4863 & 0.0 & 0.0340 & 1.8699 & 1.8334 & 1.8699 & 1.8334 \\
\hline \multirow[t]{2}{*}{$v_{c}$} & {$[3 / 1]$} & 0.0 & 0.0 & 1.6838 & 1.4914 & 0.0 & 0.0 & -1.6800 & -1.4950 \\
\hline & {$[2 / 1]^{*}$} & 0.0 & 0.0 & 1.8699 & 1.8334 & 0.0 & 0.0 & -1.8699 & -1.3591 \\
\hline \multirow[t]{2}{*}{$w_{c}$} & {$[3 / 1]$} & 0.0 & 0.0 & 0.0 & 0.0 & -0.8416 & -0.7477 & 0.8400 & 0.7480 \\
\hline & {$[2 / 1]^{*}$} & 0.0 & 0.0 & 0.0 & 0.0 & -0.9350 & -0.6796 & 0.9349 & 0.6795 \\
\hline
\end{tabular}

* Quoted from Ref. 442

TABLE II. Coordinates of the fixed points of RG equations for $N=3$ obtained within three-loop (approximant [3/1]) and two-loop (approximant [2/1]) approximations.

\begin{tabular}{|c|c|c|c|c|c|}
\hline & & 1 & 2 & 3 & 4 \\
\hline \multirow[t]{2}{*}{$u_{c}$} & {$[3 / 1]$} & 0.0 & 1.3310 & 0.0 & 0.0780 \\
\hline & {$[2 / 1]^{*}$} & 0.0 & 1.4262 & 0.0 & 0.0097 \\
\hline \multirow[t]{2}{*}{$v_{c}$} & {$[3 / 1]$} & 0.0 & 0.0 & 1.9646 & 1.8845 \\
\hline & {$[2 / 1]^{*}$} & 0.0 & 0.0 & 2.1816 & 2.1713 \\
\hline \multirow[t]{2}{*}{$w_{c}$} & {$[3 / 1]$} & 0.0 & 0.0 & 0.0 & 0.0 \\
\hline & {$[2 / 1]^{*}$} & 0.0 & 0.0 & 0.0 & 0.0 \\
\hline
\end{tabular}

* Quoted from Ref. [42] 
TABLE III. Critical exponents $\gamma$ and $\eta$ for $N=2$ calculated within three-loop (approximant [3/1]) and two-loop (approximant [2/1]) approximations.

\begin{tabular}{|c|c|c|c|c|c|c|c|c|c|}
\hline & & 1 & 2 & 3 & 4 & 5 & 6 & 7 & 8 \\
\hline \multirow[t]{2}{*}{$\gamma$} & {$[3 / 1]$} & 1 & 1.4260 & 1.3099 & 1.3360 & 1.3098 & 1.3355 & 1.3102 & 1.3357 \\
\hline & {$[2 / 1]^{*}$} & 1 & 1.4347 & 1.3218 & 1.3259 & 1.3218 & 1.3799 & 1.3218 & 1.3799 \\
\hline \multirow[t]{2}{*}{$\eta$} & {$[3 / 1]$} & 0 & 0.0257 & 0.0261 & 0.0261 & 0.0260 & 0.0261 & 0.0260 & 0.0261 \\
\hline & {$[2 / 1]^{*}$} & 0 & 0.0273 & 0.0288 & 0.0287 & 0.0288 & 0.0286 & 0.0288 & 0.0286 \\
\hline
\end{tabular}

* Quoted from Ref. [42]

TABLE IV. Critical exponents $\gamma$ and $\eta$ for $N=3$ calculated within three-loop (approximant [3/1]) and two-loop (approximant [2/1]) approximations.

\begin{tabular}{lccccc}
\hline \hline & 1 & 2 & 3 & 4 \\
\hline$\gamma$ & {$[3 / 1]$} & 1 & 1.5164 & 1.3099 & 1.3291 \\
& {$[2 / 1]^{*}$} & 1 & 1.5217 & 1.3218 & 1.3220 \\
\hline$\eta$ & {$[3 / 1]$} & 0 & 0.0238 & 0.0261 & 0.0261 \\
& {$[2 / 1]^{*}$} & 0 & 0.0246 & 0.0288 & 0.0286 \\
\hline \hline
\end{tabular}

* Quoted from Ref. [42] 